\title{
Some stress intensity factors for self-similar cracks, derived from path-independent integrals
}

\author{
FINN OUCHTERLONY \\ Swedish Detonic Research Foundation, Box 32058, S-12611 Stockholm, Sweden
}

(Received July 28, 1977)

\section{SUMMARY}

A new method of computing stress intensity factors for self-similar cracks in the plane is discussed. It is based on some known path-independent integrals whose forms make them suited for use in elastic fields showing expansional invariance. General integral formulas for the various segments of a chosen integration contour are presented and also applied. The integrals generally express the total energy release rate at self-similar growth, but for the expansion loaded star crack and the symmetrically cracked wedge opened by concentrated forces the stress intensity factors are obtained in closed form.

\section{ZUSAMMENFASSUNG}

icity.

ntent icity. ctive 'pear

nois,

$.00)$ her. lijn,

Ein neues Verfahren der Berechnung von Spannungsintensitätsfaktoren zugehörig selbstähnlichen Risssystemen in der Ebene wird diskutiert. Es beruht auf einigen bekannten wegunabhängigen Integralen, deren Formen sie in expansionsinvariablen elastischen Feldern anwendbar machen. Die allgemeinen Integralformeln der verschiedenen Abschnitte eines speziell gewählten Integrationsweges werden sowohl vorgezeigt als angewandt. Gewöhnlicherweise drücken die Integrale die totale Energiefreisetzung eines Rissystems bei selbstähnlichem Zuwachs aus. Für den expansionsbelasteten Sternriss und für den symmetrisch gespaltenen Keil, der von Punktkräften geöffnet wird, werden aber die Spannungsintensitätsfaktoren in abgeschlossener Form erhalten.

\section{Introduction}

The symmetric cracking of a plane elastic wedge by concentrated loads was studied in a previous paper by the author [1]. The analysis involved the Wiener-Hopf method, but an explicit factorization was not necessary. Thus, there should exist a more direct approach. Eshelby's work [2] shows that the $M$-integral can be used to analyze self-similar crack geometries in linearly elastic materials where the elastic field shows expansional invariance. The cracked wedge falls into this category and, indeed, by means of the $M$-integral all the results in [1] can be rederived and extended with minimal effort. Simultaneously with Eshelby's paper, Carlsson [3] 
introduced several new path-independent integrals. Of these $\bar{J}_{\boldsymbol{x}}$, which is complementary to $M$, is even better suited to the analysis. We will first discuss the shape of the contour of integration and give some formulas for special cases. Then a brief result for a pressurized crack is derived.

Internal self-similar cracks in the plane consist of a number of straight crack lines with a common origin. They fall into two overlapping categories, namely star cracks, where all the cracks have finite lengths, and cracked wedges, where two infinite radial lines form the edges. An application of $M$ or $\vec{J}_{x}$ to these cases expresses the total energy release rate at self-similar growth as a sum of a priori complicated line integrals. When the applied load consists of concentrated forces at the origin they will yield explicit results without a detailed knowledge of the elastic field. In the end, this information is sufficient to give the individual stress intensity factors in two cases. Both of them will be evaluated here to demonstrate the extreme shortness of the calculations, and they are:

1. the star crack with equally spaced cracks of the same length where, by the symmetry of loading, all crack tips possess the same values of $K_{I}$ and $\left|K_{I I}\right|$, and 2 . the cracked wedge with free edges and one symmetric crack.

These cases were analyzed in [1] for the more restricted mode $I$ situation.

\section{Basic integral formulas}

The basic formulas of the path-independent integrals in two dimensions follow from Carlsson's [3] and Eshelby's [2] papers. We will use Carlsson's notation and hence $M \equiv J_{\boldsymbol{x}}$. The defining formulas in tensorial notation are

$$
\left.\begin{array}{l}
J_{x}=\oint_{C}\left(W x_{k} n_{k}-T_{i} x_{k} u_{i, k}\right) d s, \quad \text { and } \\
\bar{J}_{x}=\oint_{C}\left(\vec{W} x_{k} n_{k}-T_{i} u_{i}-x_{k} \sigma_{i j, k} u_{i} n_{j}\right) d s .
\end{array}\right\}
$$

Here $C$ is a closed curve in a plane of linearly elastic and isotropic material. $W$ is the energy density and $\bar{W}$ is the complementary energy density which are given by $W=\bar{W}=\sigma_{i j} \varepsilon_{i j} / 2$, where $\sigma_{i j}$ denotes the stress tensor and $\varepsilon_{i j}$ the strain tensor. $n_{i}$ denotes the unit outward normal to the curve, $T_{i}=\sigma_{i j} n_{j}$ is the stress vector acting on the outside of $C$, and $u_{i}$ is the displacement vector. $d s$ denotes the length of an incremental line element along $C$.

By combining $J_{x}$ and $\bar{J}_{x}$ Carlsson [3] obtains another path-independent integral which reads

$$
I_{x}=J_{x}+\bar{J}_{x}=\oint_{C}\left[\sigma_{i j} \varepsilon_{i j} x_{k} n_{k}-T_{i} u_{i}-x_{k}\left(\sigma_{i j} u_{i}\right)_{, k} n_{j}\right] d s,
$$

and does not rest on any specific constitutive assumption. The reason for introducing it will become obvious later. 


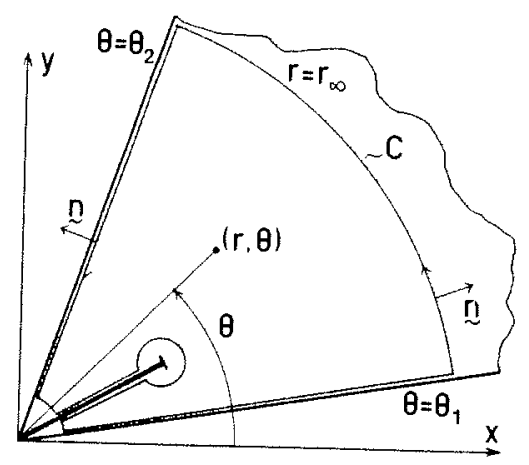

Figure 1. Cracked wedge in polar coordinate system with suggested integration contour consisting of radial line segments, centered arc segments, and a crack tip loop.

Self-similar crack geometries are conveniently described by a polar coordinate system $(r, \theta)$ which is centered at the origin of the crack lines, see Figure 1. Using a notation with physical vector components in this system, equations (2.1) and (2.2)
transform into

$$
\left.\begin{array}{l}
J_{\boldsymbol{x}}=\oint_{C}\left[\boldsymbol{W}(\boldsymbol{n} \cdot \boldsymbol{r})-\boldsymbol{r} \boldsymbol{T} \cdot \boldsymbol{u}_{, r}\right] d s, \\
\bar{J}_{\boldsymbol{x}}=\oint_{C}\left[\bar{W}(\boldsymbol{n} \cdot \boldsymbol{r})-\boldsymbol{T} \cdot \boldsymbol{u}-r \sigma_{\alpha \beta, r} u_{\alpha} n_{\beta}\right] d s, \text { and } \\
I_{\boldsymbol{x}}=\oint_{C}\left[(\boldsymbol{W}+\bar{W})(\boldsymbol{n} \cdot \boldsymbol{r})-\boldsymbol{T} \cdot(\boldsymbol{r u})_{. r}-r \sigma_{\alpha \beta, r} u_{\alpha} n_{\beta}\right] d s .
\end{array}\right\}
$$

Here $\boldsymbol{r}$ is the position vector at a point $R$ on $C, \boldsymbol{n}=\left(n_{r}, n_{\theta}\right)$ is the unit normal vector at $R, \boldsymbol{u}=\left(u_{r}, v_{\theta}\right)$ is the displacement vector, and $\boldsymbol{T}$ the stress vector at $R$. The indici $\alpha$ and $\beta$ take the "values" $r, \theta$ and the summation convention is valid. The $\sim$ denotes a vector quantity in the figures.

These expressions reduce considerably if the integration contour coincides with coordinate lines, since $n_{r}$ or $n_{\theta}=0$ along them. The energy terms vanish along radial lines since $\boldsymbol{n} \cdot \boldsymbol{r}=0$ on these. The second and third terms in all integrands above vanish along a free boundary such as the edge of a wedge or a crack surface. This makes it convenient to let $C$ consist of segments of radial lines, preferably along free boundaries, and centered circular arcs wherever possible. The only other type of segment necessary to close $C$ is the crack tip loop in Figure 1. The path-independence of our integrals implies that this contour is equivalent to any other sectionally smooth contour which can be obtained by expanding or shrinking $C$ through homogeneous material without singularities such as concentrated forces.

Likewise, the value of the integrals along an arbitrary segment $A B$ of $C$ will not change if $A B$ is replaced by a set of radial lines and centered arcs which can be 


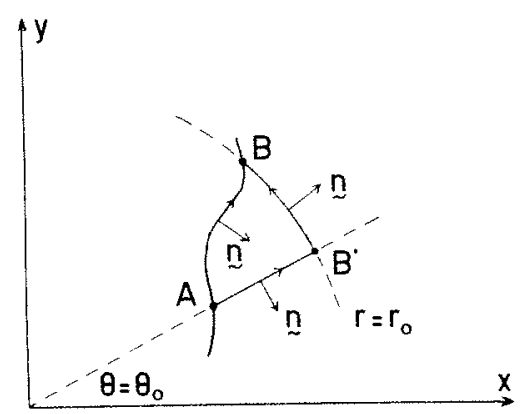

stress free are loadec crack tip according!

Figure 2. Arbitrary contour segment $A B$ with an equivalent path $A B^{\prime} B$ which consists of a radial line $A B^{\prime}$ and a centered arc $B^{\prime} B$.

shrunk onto $C$. For simplicity we regard Figure 2 with just one radial line $A B^{\prime}$ and one centered arc $B^{\prime} B$. Along $A B^{\prime}$ only the stress-displacement terms in equations (2.3) remain. With $\boldsymbol{T}=\left(-\tau_{r \theta},-\sigma_{\theta}\right), n=(0,-1)$, and $\partial f / \partial r=d f / d s$ on $A B^{\prime}$ we obtain

$$
I_{x}^{A B^{\prime}}=\int_{A B^{\prime}} \frac{d}{d s}\left[r\left(\sigma_{\theta} v_{\theta}+\tau_{r \theta} u_{r}\right)\right] d s=\left[r\left(\sigma_{\theta} v_{\theta}+\tau_{r \theta} u_{r}\right)\right]_{A}^{B^{\prime}}
$$

Along the centered $\operatorname{arc} B^{\prime} B$ also the energy term remains since $n \cdot r=r$. Employing that $\boldsymbol{T}=\left(\sigma_{r} \tau_{r \theta}\right)$ and $n=(1,0)$, that $W+\bar{W}=\sigma_{r} \varepsilon_{r}+\sigma_{\theta} \varepsilon_{\theta}+\tau_{r \theta} \gamma_{r \theta}$ both in plane strain (PD) and plane stress (PS), the strain definitions and the equations of equilibrium in polar coordinates, and that $\partial f / \partial \theta=r d f / d s$ on $B^{\prime} B$ we obtain

$$
I_{x}^{\mathbf{B}^{\prime} \mathbf{B}}=\int_{B^{\prime} \mathbf{B}} r \frac{d}{d s}\left(\sigma_{\theta} v_{\theta}+\tau_{r \theta} u_{r}\right) d s=\left[r\left(\sigma_{\theta} v_{\theta}+\tau_{r \theta} u_{r}\right)\right]_{B^{\prime}}^{B}
$$

Hence it follows that independent of the path

$$
I_{x}^{\mathrm{AB}}=I_{\boldsymbol{x}}^{\mathrm{AB}^{\prime}}+I_{\boldsymbol{x}}^{\mathrm{B}^{\prime} \mathrm{B}}=\left[r\left(\sigma_{\theta} v_{\theta}+\tau_{r \theta} u_{r}\right)\right]_{\mathbf{A}}^{\mathrm{B}} .
$$

This formal integration is possible since the path-independence of an integral implies that the integrand is a perfect differential.

An important consequence of equations (2.6) and (2.2) is that $I_{x}^{A B}$ vanishes and that

$$
J_{\boldsymbol{x}}^{\mathrm{AB}}=-\bar{J}_{\boldsymbol{x}}^{\mathrm{AB}}
$$

if both end-points of the segment $A B$ lie on radial lines which are either

I. free boundaries where $\tau_{r \theta}=\sigma_{\theta}=0$, or

II. shear free lines of symmetry where $\tau_{r \theta}=v_{\theta}=0$, or

III. fixed boundaries where $u_{\mathrm{r}}=v_{\theta}=0$, or

IV. lines where $u_{r}=\sigma_{\theta}=0$.

Equation (2.7) holds, for example, for an arbitrary crack tip loop around the tip of a

\section{Integra}

Let us divi circular aat least or: If both $t$ ? the conse?

$$
J_{\boldsymbol{x}}=\sum_{i} J_{\text {: }}
$$

when $C$

superscrip

The $\mathrm{Co}^{-}$ become

$$
\begin{aligned}
& \boldsymbol{J}_{x}^{i}=\int_{C_{r}} \\
& \bar{J}_{x}^{i}=\int_{C_{i}} !
\end{aligned}
$$

With $n$ pr Both of tr for exam

The c:

$$
\begin{aligned}
& \boldsymbol{J}_{\boldsymbol{x}}^{i}=\int_{C_{i}} \\
& \overline{\boldsymbol{J}}_{\boldsymbol{x}}^{i}=\int_{C_{i}} 1
\end{aligned}
$$

When $n$ r expressio

Using

stresses a that a crac at the dist

$$
J_{x}^{i}=-\bar{J}_{x}^{i}
$$

to the inte when $\kappa=$. 
stress free crack since both $A$ and $B$ belong to category I above. If the crack faces are loaded by a pressure of finite magnitude it holds in the limit of vanishingly small crack tip loops. Thus, if all the segments of the integration contour are chosen accordingly, we can use whichever of the integrals $J_{x}$ and $\bar{J}_{x}$ is easiest to handle.

\section{Integral formulas for contour segments}

Let us divide the integration contour into segments which are either radial lines, centered circular arcs or crack tip loops. We enumerate them consecutively $i=1,2,3, \ldots$ if at least one end-point of the segment is close to the origin and denote them then $C_{i}$. If both end-points are at infinity we use the notation $C_{\infty}$. Thus $C \hat{=} \sum_{i} C_{i}+C_{\infty}$, and the conservation laws inherent in the path-independent integrals are that both

$$
J_{x}=\sum_{i} J_{x}^{i}+J_{x}^{\infty} \text { and } \bar{J}_{x}=\sum_{i} \bar{J}_{x}^{i}+\bar{J}_{x}^{\infty}=0
$$

when $C$ encompasses homogeneous material without singularities. Here the superscript $i$ replaces $C_{i}$ and $\infty$ replaces $C_{\infty}$ for the sake of convenience.

The contributions from radial segments with $n$ oriented as on $A B^{\prime}$ in Figure 2 become

$$
\left.\begin{array}{l}
J_{x}^{i}=\int_{C_{i}}\left(\sigma_{\theta} v_{\theta, r}+\tau_{r \theta} u_{r, r}\right) r d r, \text { and } \\
\bar{J}_{x}^{i}=\int_{C_{i}}\left[\left(\sigma_{\theta}+r \sigma_{\theta, r}\right) v_{\theta}+\left(\tau_{r \theta}+r \tau_{r \theta, r}\right) u_{r}\right] d r .
\end{array}\right\}
$$

With $\boldsymbol{n}$ pointing in the other direction a minus sign enters into these expressions. Both of them vanish if $C_{i}$ belongs to any of the categories I-IV in Section 2 since, for example, $\sigma_{\theta}=0$ on a radial line implies that $\sigma_{\theta, r}=0$ there too.

The contributions from centered arcs like $B^{\prime} B$ become

$$
\left.\begin{array}{l}
J_{x}^{i}=\int_{C_{i}}\left(W-\sigma_{r} u_{r, r}-\tau_{r \theta}, v_{\theta, r}\right) r^{2} d \theta, \text { and } \\
\bar{J}_{x}^{i}=\int_{C_{1}}\left[W r-\left(\sigma_{r}+r \sigma_{r, r}\right) u_{r}-\left(\tau_{r \theta}+r \tau_{r \theta, r}\right) v_{\theta}\right] r^{2} d \theta .
\end{array}\right\}
$$

When $\boldsymbol{n}$ points inwards they change sign too. No immediate reductions of these expressions are possible.

Using the proper transformation laws and the well-known expressions for the stresses and displacements near a crack tip in local polar coordinates, we can show that a crack tip loop taken clockwise around an arbitrarily oriented crack with the tip at the distance $a$ from the origin and $n$ pointing towards the tip will contribute

$$
J_{x}^{i}=-\bar{J}_{x}^{i}=-a(1+\nu)(\kappa+1) / 4 E \cdot\left(K_{I}^{2}+K_{I I}^{2}\right)
$$

to the integral. This expression is valid both in PS when $\kappa=(3-\nu) /(1+\nu)$ and in PD when $\kappa=3-4 \nu$. By virtue of equations (2.7) and (3.2), equations (3.4) hold for an 
arbitrary loop as long as the crack surface between the end-points of $C_{i}$ and the tip is stress free. For a pressurized crack they hold for a vanishingly small loop. The equivalence between $J_{x}^{i}$ and the energy release rate for a crack with stress free surfaces was first pointed out by Budiansky and Rice [4].

$C_{\infty}$ will always consist of a centered arc. Equations (3.3) imply that if the stresses decay faster than $1 / r$, as they do when the applied load is balanced, then the contribution from $C_{\infty}$ will vanish. If an unbalanced loading is concentrated to the crack faces and to the origin, then its action far away from the crack can be reproduced by a concentrated force applied to the origin of an uncracked medium. In that case $\sigma_{r}$ and $\tau_{r \theta} \propto 1 / r$ lead to $\sigma_{r}+r \sigma_{r, r} \equiv 0$ and $\tau_{r \theta}+r \tau_{r \theta, r} \equiv 0$. Hereby the expression for $\bar{J}_{x}^{i}$ in equations (3.3) reduces to

$$
\bar{J}_{\boldsymbol{x}}^{\infty}=\int_{C_{\infty}} W r^{2} d \theta
$$

which requires less effort to evaluate than $J_{x}^{i}$ in equations (3.3). The strain energy density in equation (3.5) is given by

$$
W=\frac{1+\nu}{8 E}\left[(\kappa+1)\left(\sigma_{r}+\sigma_{\theta}\right)^{2}-8 \sigma_{r} \sigma_{\theta}+8 \tau_{r \theta}^{2}\right]
$$

valid in both PS and PD.

The stresses in an infinite wedge loaded at its point as in Figure $3 \mathrm{a}$ are given by Timoshenko and Goodier [5]. The result for a force which is directed at the angle $\phi$ with respect to the wedge bisector where $\theta=0$ is that

$$
\sigma_{r}=-\frac{2 F}{r}\left[\frac{\cos \phi \cos \theta}{2 \alpha+\sin 2 \alpha}+\frac{\sin \phi \sin \theta}{2 \alpha-\sin 2 \alpha}\right] \text { and } \sigma_{\theta}=\tau_{r \theta}=0 \text {. }
$$

Thus $\sigma_{r}$ is the only non-vanishing stress component and since $C_{\infty}$ meets the requirements of equation (2.7), equations (3.5) and (3.6) above may be combined to give

$$
\bar{J}_{x}^{\infty}=-J_{x}^{\infty}=(1+\nu)(\kappa+1) / 8 E \cdot \int_{C_{\infty}}\left(r \sigma_{r}\right)^{2} d \theta
$$

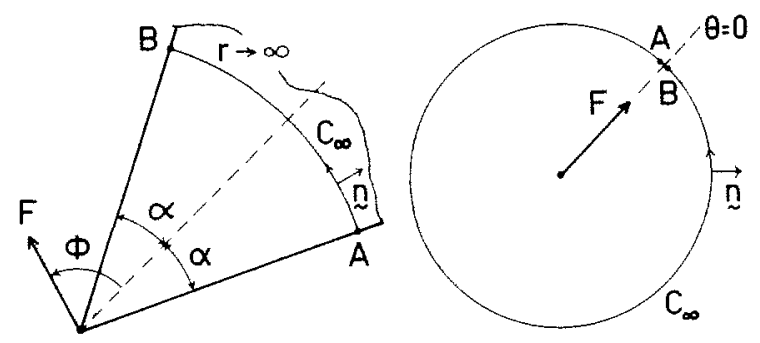

Figure $3 \mathrm{a}+\mathrm{b}$ Infinite contour segments $C_{\infty}$ for $a$ )-an infinite wedge and $b$ )-an infinite plane, both with a concentrated force applied at the origin.

in antie;

Equatis

For :

Timosfici

force $\mathrm{d}$ :

$\left\{\sigma_{r}, y\right.$

Lettin coincis" valid. I: the ress

$$
\bar{J}_{x}^{\infty}=-
$$

The fac; thus $t$ load ege

\section{App}

4.1. $P f$

The fif: star cra crack a It is $\mathrm{c}_{\mathrm{t}}$ contrit. $\tau_{\text {re }}=\mathrm{l}$. load is C.

A st:

$$
\bar{J}_{\boldsymbol{x}}^{i 1}
$$

$$
\bar{J}_{x}^{i 2}=
$$

Here supers: denot crack displas that th. 
in anticipation of an insertion of $\sigma_{r}$ from equation (3.7) for the appropriate loading. Equation (3.8) is valid both in PS and in PD.

For a point force at the origin of an infinite plane, the stress formulas of Timoshenko and Goodier [5] for PS can be extended to PD. Measuring $\theta$ from the force direction as in Figure 3b we have that

$$
\left\{\sigma_{r}, \sigma_{\theta}, \tau_{r \theta}\right\}=\frac{F}{2 \pi r}\left\{-\frac{\kappa+3}{\kappa+1} \cos \theta, \frac{\kappa-1}{\kappa+1} \cos \theta, \frac{\kappa-1}{\kappa+1} \sin \theta\right\} .
$$

Letting $C_{\infty}$ describe a whole circle, a pair of arbitrarily chosen end-points $A$ and $B$ coincide. Hence equation (2.5) implies that $\Gamma_{x}^{\infty}=0$ and that equation (2.7) again is valid. Integrating equation (3.5) with the aid of equations (3.6) and (3.9) we obtain the result

$$
\bar{J}_{x}^{\infty}=-J_{x}^{\infty}=(1+\nu) \kappa /(\kappa+1) \cdot F^{2} / 2 \pi E .
$$

The factor involving $\kappa$ above is different from those in equations (3.4) and (3.8) and thus the stress intensity factors of a star crack which is subjected to an unbalanced load generally will depend on $\nu$. This concludes the general part of our analysis.

\section{Applications}

\subsection{Pressurized star crack}

The first application of the path-independent integral $\bar{J}_{\boldsymbol{x}}$ concerns the pressurized star crack of Figure 4 . The finite part of the integration contour $C$ surrounds the crack arms with radial lines on the crack faces and vanishingly small crack tip loops. It is connected with the infinite part $C_{\infty}$ through two dashed radial lines whose contributions cancel each other. The term pressurized implies that $\sigma_{\theta}=-p$ and $\tau_{r \theta}=0$ on the crack faces, where $p$ is a pressure of constant magnitude. Hence the load is balanced and $\bar{J}_{x}^{\infty}=0$. Thus remains the contributions from the finite parts of C.

A straight application of equations (3.2) and (3.4) yields

$$
\left.\begin{array}{l}
\bar{J}_{x}^{i 1}+\bar{J}_{x}^{i 3}=-p \int_{0}^{a_{1}}\left(v_{\theta i}^{+}-v_{\theta i}^{-}\right) d r=-p V_{i}, \quad \text { and } \\
\bar{J}_{x}^{i 2}=a_{i}(1+\nu)(\kappa+1)\left(K_{I i}^{2}+K_{I I i}^{2}\right) / 4 E .
\end{array}\right\}
$$

Here index $i$ refers to the crack arm rather than the segment number. The superscripts + and - refer to the upper and lower crack faces respectively. $V_{i}\left[\mathrm{~m}^{3} / \mathrm{m}\right]$ denotes the crack volume, or gas volume, per unit transverse thickness of the $i$-th crack arm, which by definition is obtained from an integration of the crack opening displacement $\left(v_{\theta i}^{+}-v_{\theta i}^{-}\right)$. Employing the latter of equations (3.1) we obtain the result that the total crack volume is 


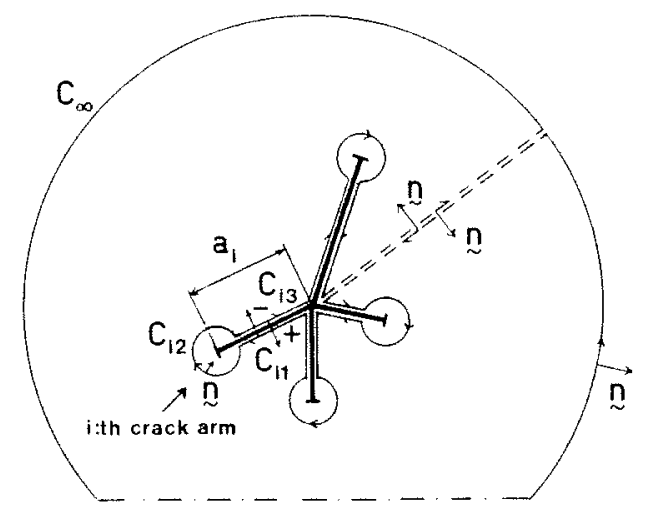

Figure 4. Pressurized star crack of arbitrary shape with matching integration contour.

$$
V=(1+\nu)(\kappa+1) / 4 p E \cdot \sum_{i} a_{i}\left(K_{I i}^{2}+K_{I r i}^{2}\right) .
$$

This result could also have been obtained through direct physical argumentation by equating the sum of the local energy release rates with the overall rate of decrease of the potential energy of the cracked body including load for a reversible quasi-static self-similar, or uniform, growth of the star crack. The final steps in such a derivation would involve Clapeyron's theorem which in our case relates the elastic energy $U[\mathrm{~J} / \mathrm{m}]$ to the crack volume through $U=p V / 2$. Equation (4.2) above also holds for a cracked wedge with pressurized crack arms.

\subsection{Wedge with a symmetric crack at the end}

The second application of $\bar{J}_{\boldsymbol{x}}$ concerns the symmetrically cracked wedge of Figure 5, where a symmetric pair of unbalanced forces of magnitude $F[\mathrm{~N} / \mathrm{m}]$ are applied at the points. The integration contour is shown in the figure. Equation (3.2) shows that all contributions from radial segments vanish, that is $\bar{J}_{\boldsymbol{x}}^{i}=0$ for odd values of $i$. Equation (3.4) gives the contribution of the crack tip loop as

$$
\bar{J}_{x}^{4}=a(1+\nu)(\kappa+1) K_{I}^{2} / 4 E,
$$

since $K_{\mathrm{Ir}}=0$ as a result of the symmetric loading. An application of equations (3.7) and (3.8) gives the contribution from $C_{\infty}$ as

$$
\bar{J}_{x}^{\infty}=(1+\nu)(\kappa+1) \cos ^{2} \phi /(2 \alpha+\sin 2 \alpha) \cdot F^{2} / E .
$$

The centered arcs $C_{2}$ and $C_{6}$ can be moved so close to the origin that the stress field very nearly is the same as in an infinite wedge loaded at its point. Equations (3.7)

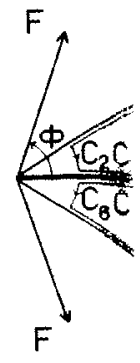

Figure 5 . wedge su pair of matching

and (3.8) ar

$$
\bar{J}_{x}^{2}=\bar{J}_{x}^{6}=
$$

where the ct is an odd $\mathrm{fu}$

Summing and rearran,

$$
K_{\mathrm{I}}=\frac{F}{\sqrt{(\pi a}}
$$

This result $w$ tends to clo:

$$
\phi<\phi_{\min }(1
$$

for outward valid when 4 combination the crack at

When the (4.3)-(4.5) cl equations ( 3 .

$$
\bar{J}_{\boldsymbol{x}}^{\infty}=(1+\nu
$$

Since $K_{I} \mathrm{mu}$ 


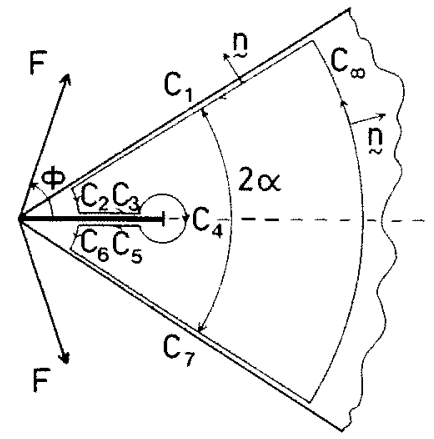

Figure 5. Symmetrically cracked wedge subjected to a symmetric pair of unbalanced forces with matching integration contour.

and (3.8) are thus valid here too. With $2 \alpha \rightarrow \alpha$ and $\phi \rightarrow \pm(\phi-\alpha / 2)$ they yield

$$
\bar{J}_{x}^{2}=\bar{J}_{x}^{6}=-(1+\nu)(\kappa+1) F^{2} / 4 E \cdot\left[\frac{\cos ^{2}(\phi-\alpha / 2)}{\alpha+\sin \alpha}+\frac{\sin ^{2}(\phi-\alpha / 2)}{\alpha-\sin \alpha}\right],
$$

on by ase of -static vation nergy ds for

where the cross-product term from the squaring of equation (3.7) vanishes because it is an odd function of $\boldsymbol{\theta}$.

Summing all these contributions, equating the sum with zero as in equations (3.1), and rearranging we obtain the opening mode stress intensity factor as

$$
K_{I}=\frac{F}{\sqrt{(\pi a)}}\left(\sin \phi-\cos \phi \frac{2 \sin ^{2} \alpha}{2 \alpha+\sin 2 \alpha}\right) \sqrt{\left[\pi(2 \alpha+\sin 2 \alpha) /\left(\alpha^{2}-\sin ^{2} \alpha\right)\right]} .
$$

This result was derived by the author in [1]. Equation (4.6) implies that the crack tip tends to close when

Ire 5 , ed at $s$ that of $i$.

field

$$
\phi<\phi_{\min }(\alpha)=\operatorname{atan}\left[2 \sin ^{2} \alpha /(2 \alpha+\sin 2 \alpha)\right],
$$

for outward pointing forces, that is $0 \leq \phi \leq \pi$. Strictly seen equation (4.6) is only valid when $\phi \geq \phi_{\min }$. The range of its validity is extended to $\phi<\phi_{\min }$ if it is used in combination with some other loading and the joint action results in an opening of the crack at its tip. $\phi_{\min }(\alpha)$ is plotted in Figure 6.

When the wedge is loaded only by the upper force in Figure 5, equations (4.3)-(4.5) change. $\bar{J}_{x}^{6}$ vanishes but $\bar{J}_{x}^{2}$ retains its value. $\bar{J}_{x}^{4}$ will contain the $K_{I I}^{2}$ term of equations (3.4) since $K_{I I}$ no longer vanishes. The contribution from $C_{\infty}$ now becomes

$$
\bar{J}_{x}^{\infty}=(1+\nu)(\kappa+1) F^{2} / 4 E \cdot\left[\frac{\cos ^{2} \phi}{2 \alpha+\sin 2 \alpha}+\frac{\sin ^{2} \phi}{2 \alpha-\sin 2 \alpha}\right] .
$$

Since $K_{I}$ must be exactly half the value given by equation (4.6), calculations yield the 


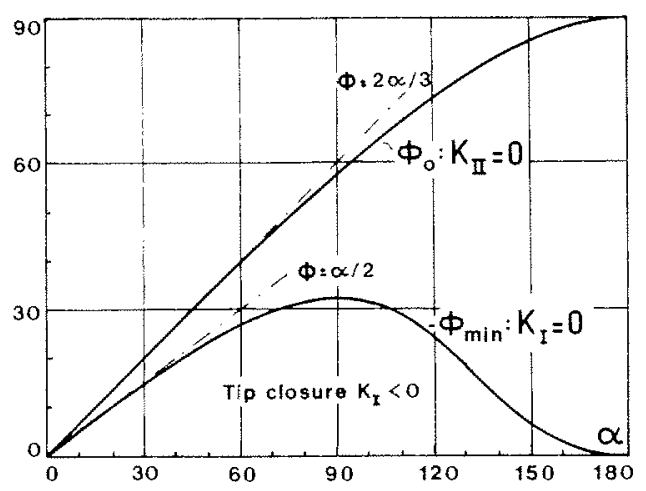

Figure 6. Angles of force application relative to the crack of a symmetrically cracked wedge which cause tip closure $\left(\phi_{\min }\right)$ when one or two forces are applied and in-plane crack extension $\left(\phi_{0}\right)$ when only one force is present.

stress intensity factors

$$
\left.\begin{array}{l}
K_{I}=\frac{F}{\sqrt{(\pi a)}} \cdot \frac{1}{2}\left(\sin \phi-\cos \phi \frac{2 \sin ^{2} \alpha}{2 \alpha+\sin 2 \alpha}\right) \sqrt{\left[\pi(2 \alpha+\sin 2 \alpha) /\left(\alpha^{2}-\sin ^{2} \alpha\right)\right]} \\
K_{I I}=\frac{F}{\sqrt{(\pi a)}} \cdot \frac{1}{2}\left(\cos \phi-\sin \phi \frac{2 \sin ^{2} \alpha}{2 \alpha-\sin 2 \alpha}\right) \sqrt{\left[\pi(2 \alpha-\sin 2 \alpha) /\left(\alpha^{2}-\sin ^{2} \alpha\right)\right]} .
\end{array}\right\}
$$

With a change of sign for $K_{I I}$, equations (4.9) give the results for the lower force too. The expression for $K_{I I}$ will become zero when

$$
\phi=\phi_{0}(\alpha)=\operatorname{atan}\left[(2 \alpha-\sin 2 \alpha) / 2 \sin ^{2} \alpha\right] .
$$

A force attacking in this direction would cause the crack to extend in its own plane. $\phi_{0}(\alpha)$ is also plotted in Figure 6 .

\subsection{Expansion loaded star crack}

The third application of $\vec{J}_{x}$ concerns the expansion loaded star crack which has $n \geq 2$ arms of equal lengths $a$ as is shown in Figure 7. The forces act at the origin. They are directed along the crack bisectors and thus balanced. Because of the symmetry we use another integration contour than that suggested by Figure 4, namely one corresponding to a symmetrically cracked wedge with normally constrained edges. The crack bisectors, which form these edges and where $\tau_{r \theta}=v_{\theta}=0$, belong to category II in Section 2. Hence equation (3.2) shows that all contributions from radial segments vanish, thus $\bar{J}_{x}^{i}=0$ when $i$ is odd. The crack tip loop contribution $\bar{J}_{x}^{4}$ is the same as in equation (4.3) since $K_{I I}=0$ and the balanced loading implies that $\bar{J}_{x}^{\infty}=0$. A direct application of equations (3.7) and (3.8) with $\phi=0$ give the 


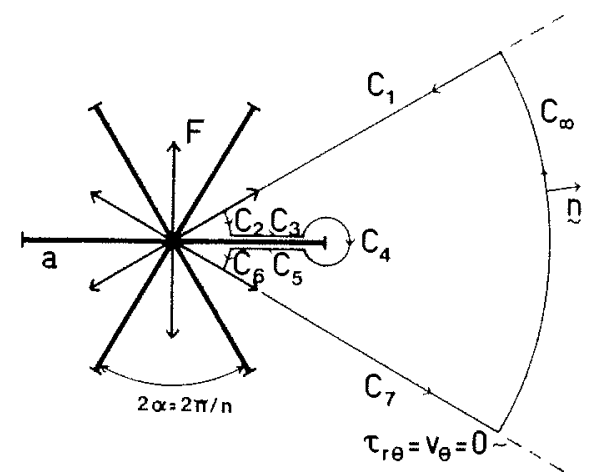

Figure 7. Expansion loaded star crack with arms of equal lengths and matching integration contour.

contributions from $C_{2}$ and $C_{6}$ as

$$
\bar{J}_{x}^{2}=\bar{J}_{x}^{\dagger}=-(1+\nu)(\kappa+1) /(2 \alpha+\sin 2 \alpha) \cdot F^{2} / 8 E .
$$

Hereby the contributions from all segments are known.

A straight-forward application of equation (3.1) yields the opening mode stress intensity factor

$$
K_{I}=\frac{F}{\sqrt{(\pi a)}} \cdot \sqrt{[\pi /(2 \alpha+\sin 2 \alpha)]},
$$

where $\alpha=\pi / n$ for a star crack but may assume any value $\alpha \leq \pi$ for a wedge. This result was derived by the author in [1] and [6].

When the number of crack arms is even and $n \geq 4$, and in addition only every plane. second force in Figure 7 is present, then $\bar{J}_{x}^{6}=0$ and $\bar{J}_{x}^{2}$ retains its value or vice versa: $\bar{J}_{x}^{4}$ will again contain the $K_{I I}^{2}$ term and as a consequence

$$
K_{I}=\left|K_{I I}\right|=\frac{F}{\sqrt{(\pi a)}} \cdot \frac{1}{2} \sqrt{[\pi /(2 \alpha+\sin 2 \alpha)]},
$$

$n \geq 2$

They

metry y one edges. ng to from ion $\bar{J}_{x}^{4}$ is that

with the sign of $K_{I I}$ alternating from tip to tip. Proceeding in counter-clockwise direction, $K_{I I}$ is positive at a tip situated before a loaded sector.

The plane crack in Figure 8 is a star crack with $n=2$ or $\alpha=\pi / 2$. When a pair of balanced forces are applied at the origin equation (4.12) holds and we get the well-known result $K_{I}=F / \sqrt{(\pi a)}$. When only the upper force is applied the load is no longer balanced and the contribution from $C_{\infty}$ cannot be neglected. The integration contour is shown in Figure 8. The contributions from the two dashed connections cancel each other and those from $C_{1}, C_{2}, C_{4}, C_{6}$ and $C_{8}$ vanish because of the stress free crack faces. After an application of equations (3.4), (3.10), (3.8) and (3.7) with 


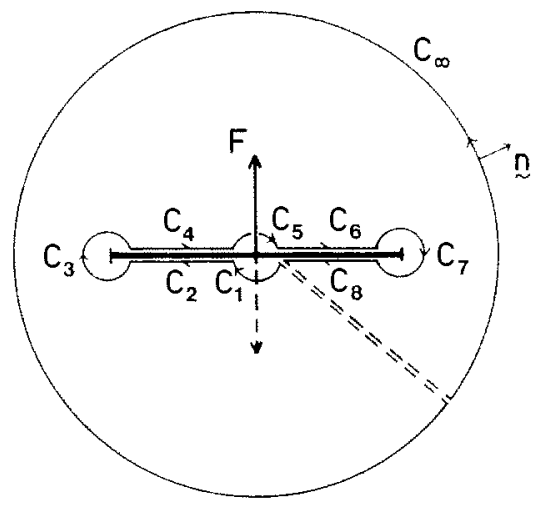

REFEREN

[1] Ouchter (1977) 1

[2] Eshelby C. Sih e

[3] Carlsson Principle

[4] Budians (1973) ?

[5] Timosh:

[6] Ouchte: (1976) \&

Figure 8. Plane crack with one concentrated force applied at the midpoint and matching integration contour.

$\phi=0$ and $\alpha=\pi / 2$ there remain

$$
\left.\begin{array}{l}
\bar{J}_{x}^{3}=\bar{J}_{x}^{7}=a(1+\nu)(\kappa+1)\left(K_{I}^{2}+K_{I D}^{2}\right) / 4 E, \\
\bar{J}_{x}^{\infty}=(1+\nu) \kappa /(\kappa+1) \cdot F^{2} / 2 \pi E, \quad \text { and } \\
\bar{J}_{x}^{5}=-(1+\nu)(\kappa+1) F^{2} / 4 \pi E .
\end{array}\right\}
$$

Since $K_{I}$ for this case is exactly half of what the case with balanced forces gives, equations (3.1) and (4.14) yield the stress intensity factors

$$
K_{I}=\frac{F}{2 \sqrt{(\pi a)}} \text { and }\left|K_{I I}\right|=\frac{F}{2 \sqrt{(\pi a)}} \cdot\left(\frac{\kappa-1}{\kappa+1}\right) \text {. }
$$

$K_{I I}$ is negative for the right-hand crack tip and positive for the left-hand one. If the upper force is turned $90^{\circ}$ clockwise there results for the right-hand tip

$$
K_{I I}=\frac{F}{2 \sqrt{(\pi a)}} \text { and } K_{I}=\frac{F}{2 \sqrt{(\pi a)}} \cdot\left(\frac{\kappa-1}{\kappa+1}\right)
$$

which is valid for the left-hand tip too with changed sign for $K_{I}$. Equations (4.15) and (4.16) are special cases of a result derived by Sih et al. [7], which takes into account the case when the crack arms have different lengths. Neither the information contained in the $\bar{J}_{x}$-integral, nor the one in the $J_{x}$-integral, suffice to resolve the stress intensity factors in that situation.

\section{Acknowledgements}

The author would like to thank Dr. Fred Nilsson at the Royal Institute of Technology (KTH) for the valuable discussion which initiated this work, and Prof. Janne Carlsson at KTH for helpful suggestions on the manuscript. The Swedish Board for Technical Development (STU) financed this work in cooperation with Swedish industry. 
REFERENCES

[1] Ouchterlony, F., Symmetric Cracking of a Wedge by Concentrated Loads. Int. J. Engng. Sci. 15

[2] Eshelby, J. D., The Calculation of Energy Release Rates, in Prospects of Fracture Mechanics, eds. G. C. Sih et al., Noordhoff, Leyden 1974, p. 69.

[3] Carlsson, J., Path Independent Integrals in Fracture Mechanics and their Retation Principles, Ibid p. 139.

[4] Budiansky, B. and Rice, J. R., Conservation Laws and Energy Release Rates. J. Appl. Mech. 40 (1973) 201.

[5] Timoshenko, S. and Goodier, J. N., Theory of Elasticity, McGraw-Hill, N.Y. 1951, pp. 97 and 113 [6] Ouchterlony, F., Stress Intensity Factors for the Expansion Loaded Star Crack. Engng. Fract. Mech. 8
(1976) 447.

[7] Sih, G. C., Paris, P. C. and Erdogan, F. Crack Tip Stress Intensity Factors for Plane Extension and Plate Bending Problems. J. Appl. Mech. 29 (1962) 306. 
